\title{
ORIGINAL
}

\section{FACTORES DE RIESGO CARDIOVASCULAR EN LA REGIÓN DE MURCIA, ESPAÑA *}

\author{
María José Tormo Díaz (1), Carmen Navarro Sánchez (1), María Dolores Chirlaque López (1) y \\ Domingo Pérez Flores (2). \\ (1) Servicio de Epidemiología. Consejería de Sanidad y Política Social de la Región de Murcia. \\ (2) Departamento de Bioestadística. Facultad de Medicina de Murcia. \\ $\left({ }^{*}\right)$ Este trabajo se ha financiado parcialmente con una ayuda a la investigación FIS (expediente número 92/0902).
}

\section{RESUMEN}

Fundamento: La Región de Murcia es un ánea de alta mortalidad coronaria y cenebrovascular en el contexto español. Además. la tendencia en mortalidad coronaria, descendente en prácticamente todas las áreas geográficas españolas, se ha incrementalo en ésta ligcramente durante el periodo 1985-1991. En este estudio se evahían las prevalencias poblacionales de difenentes factores de riesgo cardiovascular en la Región de Murcia

Métodas: El trabajo se ha realizado mediante una encuesta a una población muestral representativa de la poblaciởn adukta de la región (18-65 años). en la que se chtuvo una tasa de respuesta vel 61\%. Se realizó medición estandarizada de la vensión anterial. de la obesidad y de los lípicios séricos. junio a la aplicación de un chestionario sobre consumo de tahaco. actividad fisica y diabetes. Presentación de datos estantariradks ghobales y truncakos pess los grupos de edad de 35-64 años. La recogida de información se ikvó a cabo desce noviembre de 1991 a narzi de 1993.

Resultodos: La prevalencia de consumo de tatbaco nesultante es de 54,40 en twoubres y $31,3 \%$ en mujeres. Las cilras de hipereensioun son superiores en los hombres 132.34 preva-

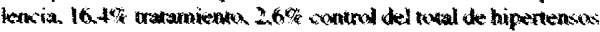

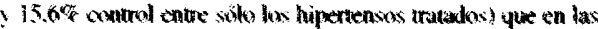

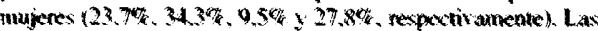
cifres medias de colesternl son bajas en ambus wexus aci como

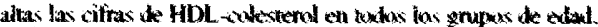

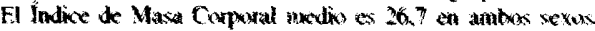
aunque las mujeres presentan una mayor variabilidiad. La pre-

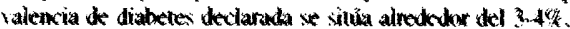

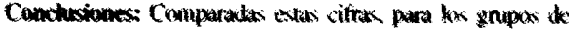

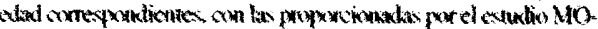

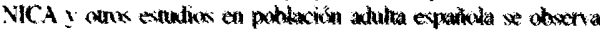
que. salno el eievadk consumo de tabaco y el allo indice de

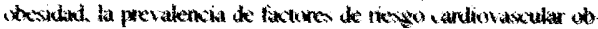

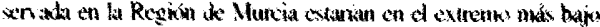

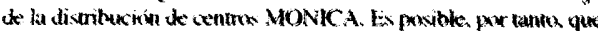
existan diferencias en la asistencia al paciente conomano que ex. pliyuen en parte las tenkerkizs destatomables en momalidad coro. naria obsernadas en la Region de Murcia

Palabras clave: Prevalencia. Encuesta. Factores de rieszo. Enfermediades candiotasculares. Hipertension. Obesitad. Ta baco. Lipidos plasmaticess. Actividad fisica.

Correspondencia

María José Torno Día

Servicio de Epidemiolugía

Avda. Ronda de Levante 11

30008 Murcia

Fax. 968201614

\section{ABSTRACT}

Background: The Region of Murcia is an area. within the Spanish context, of high coronary and stroke monality. Moreover, the trend in ischaemic heart disease mortality. decreasing for almost all geographical Spanish areas, has sulfered in Murcia a slight increase during the period 1985-1991. In this study the population prevalences to different cardiovascular risk factors axe evaluated.

Methods: Survey of a random sample of actult population (aged $18-65$ ) with a response rate of $61 \%$. Standardised measurement of anterial blood pressure, obesity and senum lipids besides a questionnaire of tobacco consunne. physical activity and diabetes. Presentation of standantised results for overall age groups and for the truncated $35-04$ sge group. The feld work were from nowember 1991 to manch 1993.

Results: Totwowo smoking prevalence rives up to $54,4 \%$ in men and $31,3 \%$ in nomen. Figures for hypertetavin are tess

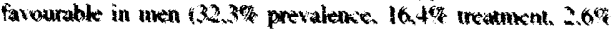
hypertension control among all hypertensives and $15.6 \%$ crut and anong anly treated hypertencises) that anom nommen

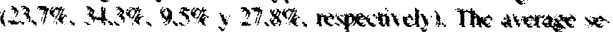
rum concentrations of chalesterol are kin in thoth wexen as hish are the HDL. sholesterol concentrations. Mean Bedh Mas Index

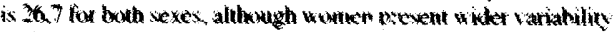

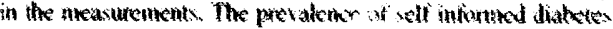
is around $3.4 \%$

Cowlusions: Comparing the higures for the compenpon ding age groups. with thuse offered by the MONKC A stwdy and with other stodiex on Spanich adull poputation it is pousible to

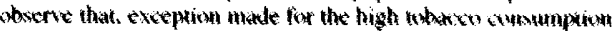
and Body Mass Index seet in the Region of Nukcia, the mefal!

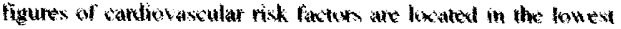
end of the distribution of MONICA centres ranked axording their pretalence of cardiovascular rish fackon. II might exist. therefore, differences in the management of the conchaty patient that could explain an least put of the unfacourable conomary heart mortality trends observed in the Region of Murcia.

Key words: Prevalence, Survey, Risk Fitchor. Cardiovascular Diseases. Hypertension. Obexity. Tobatoro. Sertm lipids. Physical activity. 


\section{INTRODUCCIÓN}

La Región de Murcia presenta una de las mayores tasas estandarizadas de mortalidad por cardiopatía isquémica y cerebrovascular entre las Comunidades Autónomas (CCAA) españolas ${ }^{1}$. Respecto a la cardiopatía isquémica, su situación empeoró en el período 1985-1991, pasando de ocupar el sexto puesto entre las CC.AA. con mortalidad más elevada, a situarse en segunda posición. Esta situación se ha traducido además, en un incremento absoluto en la mortalidad coronaria. Así, durante el mismo período, la tasa de incremento anual ha sido de $1,35 \%$ cuando el resto de las CC.AA., excepto Galicia, presentaban reducciones netas. Estos datos confirman anteriores estudios de tenden$\operatorname{cias}^{2,3}$, en los que a lo largo de sucesivos años se observaba que la tendencia decreciente en la mortalidad por cardiopatía isquémica observada en España no se materializaba en esta región. En cuanto a la mortalidad cerebrovascular, las tasas estandarizadas en 1991 fueron las más elevadas del Estado español, aunque se observó una tendencia decreciente, similar al promedio, confirmado con anterioridad por los estudios mencionados ${ }^{2,3}$.

Las modificaciones en las tasas de mortalidad por cardiopatía isquémica y enfermedad cerebrovascular se pueden deber al cambio en la exposición poblacional de factores de riesgo y protectores o a cambios en la asistencia, diagnóstico y terapéutica médica. Mientras de estos últimos aspectos no existe prácticamente información, de los primeros existía información fragmentaria. Así, se tenían, desde 1982, estimaciones de la prevalencia de hipertensión y de su nivel de control, siendo la primera elevada $(26 \%)^{4}$ y el segundo casi inexistente $(5 \%$ de hipertensos controlados $)^{5}$. La Encuesta $\mathrm{Na}$ cional de Salud señalaba un elevado consumo de tabaco entre los hombres así como un generalizado sedentarismo en las mujeres ${ }^{6}$. Prácticamente no existían datos poblacionales sobre las concentraciones lipídicas séricas ni sobre obesidad. El estudio que aquí se presenta aporta información sobre la prevalencia de los principales factores de riesgo cardiovascular en una región española de alta mortalidad coronaria y cerebrovascular.

\section{POBLACIÓN Y MÉTODOS}

Se seleccionó una muestra representativa de la población adulta de Murcia de 18-65 años a la que se invitó a participar a través de carta, llamada telefónica y visita a domicilio. La muestra, aleatoria y polietápica, se obtuvo después de definir cuotas muestrales según área de salud, tipo de residencia, grupos de edad y sexo. Para evitar una reducción en la precisión del estudio debido al problema de las no respuestas se sobremuestreó seleccionándose a 5.087 personas para una precisión deseada de $\pm 1.9 \%$, un error alfa de $5 \%$ y una prevalencia esperada del $50 \%$ (para el factor de riesgo supuestamente más prevalente: tabaco). El trabajo de campo fue llevado a cabo desde noviembre de 1991 a marzo de 1993.

Las mediciones de la tensión arterial se realizaron mediante esfigmomanómetros de mercurio previamente calibrados (modelo Riester-Diplomat 660/306, Alemania) siguiendo el protocolo $\mathrm{MONICA}^{7}$, considerando la tasa arterial sistólica (TAS) y diastólica (TAD) como la media de dos lecturas consecutivas de los sonidos $1 .^{\circ}$ y $5 .^{\circ}$ de Korotkoff, respectivamente. Las mediciones se realizaron después de la entrevista, en el brazo derecho, con la persona sentada y sin ayunar, registrándose la temperatura ambiente.

El protocolo MONICA hace un especial énfasis en el entrenamiento, evaluación y seguimiento de las personas que van a medir la tensión arterial. Por este motivo se utilizó un vídeo del programa americano HDFP (Hypertension, Detection and Follow up Progam, Houston, TX) donde se registran diferentes sonidos de Korotkoff al mismo tiempo que en la pantalla aparece la columna de mercurio descendiendo, permitiendo rea- 
lizar la lectura simultánea e independiente a varios observadores. De estos sonidos, siete eran idénticos. De esta manera se tenía información suficiente para evaluar la variabilidad intraobservador e interobservador. Una vez al mes, durante el curso de entrenamiento, se fue pasando el vídeo hasta el final del trabajo de campo. Por tanto, sc contaban con varias evaluaciones de cada observador, permitiendo monitorizarles a lo largo del tiempo. En cada ocasión, se comparaba la desviación entre la medición del observador y la ofrecida por los productores del vídeo como correcta y esta diferencia se evaluaba con respecto al máximo grado de desviación aceptable por parte del observador para que la precisión global del estudio no se modificara. El diseño y la evaluación de los seis primeros observadores han sido publicados con anterioridad ${ }^{8}$. La desviación de la TAS se mantuvo por debajo del límite establecido desde el principio, mientras que la correspondiente a la TAD sobrepasó el límite al principio del entrenamiento para decrecer posteriormente.

Dado el momento actual de transición en la clasificación de la hipertensión, se ha seguido una doble vía para el análisis y presentación de resultados. Por una parte, siguiendo las recomendaciones recientes del americano Joint National Committee on Detection, Evaluation and Treatment of high blood pressure ${ }^{9}$, se ha utilizado como definición de hipertenso a toda aquella persona con $\mathrm{TAS} \geq 140$ ó $\mathrm{TAD} \geq 90 \mathrm{mmHg}$ y/ó con tratamiento médico farmacológico hipotensor. Por otra parte, siguiendo la clasificación de la OMS $^{10}$ utilizada en el estudio MONICA se ha considerado como hipertensa aquellas personas con TAS $\geq 160 \mathrm{~mm}$. Hg. ó $\mathrm{TAD} \geq 95 \mathrm{~mm}$. Hg. y/ó con tratamiento médico farmacológico hipotensor. Esta clasificación es conveniente para poder comparar los resultados de nuestro estudio con los proporcionados por los 40 centros internacionales que componen el estudio MONICA. Considerando la primera clasificación, junto a la prevalencia se ha calculado la proporción de personas hipertensas tratadas farmacológicamente, el porcentaje de hipertensos totales controlados, el porcentaje de hipertensos tratados farmacológicamente que están controlados y el porcentaje de hipertensos conocidos (personas a las que un médico les había dicho alguna vez que tenían la TA elevada). La segunda clasificación se ha utilizado para calcular el porcentaje de hipertensos observados en la medición física de la TA, considerados como aquellas personas con TAS $\geq 160$ $\mathrm{y} / \mathrm{o} \mathrm{TAD} \geq 95 \mathrm{mmHg}$, el porcentaje de hipertensos reales (equivalente al grupo "actual hypertension" del MONICA ${ }^{11}$ ) que serían aquellas personas con $\mathrm{TAS} \geq 160 \mathrm{y} / \mathrm{o}$ $\mathrm{TAD} \geq 95 \mathrm{mmHg}$ y/o tratamiento farmacológico. De estos dos grupos se puede calcular el grado de control de la hipertensión: prevalencia (\%) de la hipertensión real menos la prevalencia de la hipertensión observada (\%) partido por la prevalencia de hipertensión real (\%). La tasa de hipertensión real de la OMS sería la equivalente a la tasa de hipertensión definida de la clasificación del Joint National Committee aunque con diferente punto de corte.

El cuestionario, estructurado y administrado por entrevistadores previamente entrenados, recogió información adicional sobre antecedentes de enfermedad cardiovascular, consumo habitual de tabaco (considerando fumador habitual a aquella persona que fumaba al menos 1 cigarrillo al día y ex-fumador a aquella persona fumadora habitual en el pasado pero sin consumo en los seis meses anteriores a la entrevista), y un cuestionario de actividad física validado ${ }^{12}$ en ambos sexos que medía la duración y el consumo energético de diferentes actividades físicas ocupacionales y de tiempo libre.

El cuestionario también contenía preguntas sobre si alguna vez un médico le había dicho que tenia la glucosa alta en sangre o que era diabético y si estaba haciendo algún tipo de tratamiento (dietético y/o farmacológico) por este motivo. En base a esta información se ha clasificado a la persona como diabética en el caso de que tuviera un diag- 
nóstico médico previo y estuviera haciendo algún tipo de tratamiento. Se calcularon también las proporciones de diabéticos que utilizan insulina, antidiabéticos orales y los que estaban bajo dieta de forma exclusiva.

Se midieron la altura y el peso para calcular el Índice de Masa Corporal (IMC) y, siguiendo el protocolo MONICA se considera obesas a aquellas personas con $I M C \geq 30$. Se extrajo una muestra de sangre en ayuno de al menos 12 horas, determinando el nivel sérico de colesterol por medio de métodos enzimáticos (coeficiente de variación de $3.8 \%$ y $2.96 \%$ para el control normal y el alto, respectivamente). Se ha considerado con hipercolesterolemia límite a aquellas personas que presentaron concentraciones entre 200 y $249.9 \mathrm{mg} / \mathrm{dl}$ e hipercolesterolémicas a aquellas personas con niveles $\geq 250 \mathrm{mg} / \mathrm{dl}^{13}$.

El Índice de Masa Corporal se calculó en base a la talla y al peso medidos con tallímetro y báscula calibrada según la formula $\mathrm{IMC}=$ peso $(\mathrm{kg}$.$) / talla (\mathrm{m})^{2}$.

Para estimar las prevalencias se utilizaron pesos muestrales que tienen en consideración el diseño muestral y la tasa de no respuesta ${ }^{14}$. Estos pesos permitcn la corrección de las tasas a nivel de las variables diseño (sexo, edad, área sanitaria y tipo de residencia).

Las prevalencias se presentan por grupos de edad decenales (excepto el primero que va de 18-24 años), tanto globales crudas como estandarizadas por la población mundial estándar, para permitir la comparabilidad con otros estudios. También se presentan, estandarizadas, las prevalencia truncadas para el grupo de 35-64 años de edad, que permite una comparación directa con las cifras editadas por el estudio MONICA ${ }^{1 !}$.

\section{RESULTADOS}

La muestra elegible ha sido de 5.087 personas con una tasa de respuestas al cuestio- nario del $61 \%$ (3.091 personas). La tasa de respuesta obtenida para el cuestionario y el examen físico fue del $61 \%$. El $16 \%$ de las no respuestas fueron negativas a participar y el resto a la imposibilidad de localizar a las personas después de varios intentos $(17 \%) \mathrm{u}$ otros motivos $(6 \%)$. Se realizó una llamada telefónica a una muestra aleatoria de las no respuesta con teléfono $(n=347)$. El análisis indicó una menor frecuencia de diagnóstico clínico de hipertensión en este grupo. Un $78 \%$ ( 2.420 personas) de las personas entrevistadas proporcionó una muestra de sangre para el análisis de lípidos plasmáticos.

El consumo habitual de tabaco es del $54,4 \%$ en los hombres y del $31,3 \%$ en las mujeres (tabla 1). En los hombres, el consumo es elevado en todas las edades mientras en las mujeres las tasas son muy elevadas en los grupos más jóvenes para disminuir en los más avanzados. El consumo medio de cigarrillos es de 18,4 cigarrillos/día en los hombres y 11,8 cigarrillos/día en las mujeres. La proporción de ex-fumadores aumenta progresivamente en los hombres, siendo globalmente de un $11,8 \%$. Las mujeres presentan una mayor tasa de ex-fumadoras en los grupos de edad con mayor consumo, siendo globalmente la prevalencia de ex-fumadoras del $3,3 \%$.

Las TAS y TAD medias aumentan linealmente con la edad, siendo más elevadas ambas en los hombres para cualquier grupo de edad, aunque las diferencias tienden a decrecer con ésta, y prácticamente se igualan en el grupo de 55-65 años (tabla 2). La TAS media en hombres es de $130,8 \mathrm{mmHg}$ y $123,3 \mathrm{mmHg}$ en las mujeres. Utilizando el criterio del Joint National Committee ${ }^{9}$ el $32,3 \%$ de los hombres y el $23,7 \%$ de las mujeres pueden ser clasificados como hipertensos. Las diferencias por grupos de edad son muy importantes en las edades más jóvenes, donde los hombres superan a las mujeres a expensas de sus cifras de TAS más elevadas. Este patrón se invierte en el grupo de 45-54 años, para ser más frecuente la hipertensión en las mujeres de edad más 
Tabla 1

Prevalencia (\%) y consumo medio (desviación estándar) de tabaco según grupos de edad y sexo

\begin{tabular}{|c|c|c|c|c|c|c|c|c|}
\hline Hombres & $\begin{array}{c}18-24 \\
n=176\end{array}$ & $\begin{array}{l}25-34 \\
n=429\end{array}$ & $\begin{array}{c}35-44 \\
n=352\end{array}$ & $\begin{array}{c}45-54 \\
n=353\end{array}$ & $\begin{array}{l}55-65 \\
n=333\end{array}$ & $\begin{array}{c}\text { Tasa } \\
\text { población } \\
18-6.5 \\
n=1.513\end{array}$ & $\begin{array}{c}\text { Tasa pobl. } \\
\text { estandar. } \\
\quad 18-6.5 \\
n=1.513\end{array}$ & $\begin{array}{c}\text { Tasa } \\
\text { truncada } \\
\text { estandar. } \\
35-64 \\
n=869\end{array}$ \\
\hline $\begin{array}{l}\text { Fumadores } \\
\text { habituales (\%) }\end{array}$ & 57,9 & 62,3 & 54,9 & 48,2 & 44,7 & 54,4 & 54,0 & 49,9 \\
\hline $\begin{array}{l}\text { Consunto medio } \\
\text { en fumadores (DE) }\end{array}$ & $\begin{array}{l}13,7 \\
(6,7)\end{array}$ & $\begin{array}{r}17,3 \\
(9,7)\end{array}$ & $\begin{array}{c}21,1 \\
(11,6)\end{array}$ & $\begin{array}{l}21,0 \\
(9,7)\end{array}$ & $\begin{array}{c}20,6 \\
(10,7)\end{array}$ & $\begin{array}{c}18,4 \\
(10,3)\end{array}$ & 18,8 & 20.9 \\
\hline Ex-fumadores $(\%)$ & 4,07 & 7,7 & 12,7 & 13,4 & 20,9 & 11,8 & 12,6 & 15,7 \\
\hline
\end{tabular}

\begin{tabular}{|c|c|c|c|c|c|c|c|c|}
\hline Mujeres & $\begin{array}{c}18-24 \\
n=178\end{array}$ & $\begin{array}{c}25-34 \\
n=4.35\end{array}$ & $\begin{array}{c}35-44 \\
n=357\end{array}$ & $\begin{array}{c}45-54 \\
n=294\end{array}$ & $\begin{array}{c}55-65 \\
n=313\end{array}$ & $\begin{array}{c}\text { Tasa } \\
\text { población } \\
18-65 \\
n=1.577\end{array}$ & $\begin{array}{c}\text { Tasa pobl. } \\
\text { estandar. } \\
18-65 \\
n=1.577\end{array}$ & $\begin{array}{c}\text { Tasa } \\
\text { truncada } \\
\text { estandar. } \\
18-65 \\
n=920\end{array}$ \\
\hline $\begin{array}{l}\text { Fumadoras } \\
\text { habituales }(\%)\end{array}$ & 53,5 & 52,0 & 37,2 & 7,4 & 4,0 & 31,3 & 29,6 & 15,9 \\
\hline $\begin{array}{l}\text { Consumo medio } \\
\text { en fumadoras (DE) }\end{array}$ & $\begin{array}{l}10,7 \\
(6,7)\end{array}$ & $\begin{array}{l}12,2 \\
(6,8)\end{array}$ & $\begin{array}{l}11,5 \\
(7,2)\end{array}$ & $\begin{array}{l}16,6 \\
(10,8)\end{array}$ & $\begin{array}{l}11,6 \\
(7,6)\end{array}$ & $\begin{array}{l}11,8 \\
(7,0)\end{array}$ & 11,9 & 12,1 \\
\hline Ex-fumadoras $(\%)$ & 3,9 & 4,4 & 4,8 & 1,4 & 1,3 & 3,3 & 3.5 & 3,1 \\
\hline
\end{tabular}

' Estandarizada por la población mundial: DE: desviación estándar.

avanzada. Es dos veces más frecuente que una mujer hipertensa csté bajo tratamiento farmacológico que un hombre hipertenso $(34,3 \%$ vs $16,4 \%$, respectivamente). El grado de control de la hipertensión poblacional, considerando esta clasificación ${ }^{9}$, es también muy diferente entre sexos $(9,5 \%$ de las mujeres hipertensas estarían controladas mientras que tan solo lo estarían el 2,6\% de los hombres). Las diferencias se mantienen en el grado de control de la población hipertensa tratada: mientras el $27,8 \%$ de las mujeres hipertensas tratadas farmacológicamente están controladas tan solo lo están el $15,6 \%$ de los hombres de su misma categoría. Por último, más mujeres $(54,9 \%)$ que hombres $(40 \%)$ han recibido por parte de su médico la información de que tienen la tensión arterial elevada.

Utilizando las categorías utilizadas por el estudio MONICA" (tabla 3), la prevalencia de hipertensión observada es del $10,1 \%$ en hombres y de $8,7 \%$ en mujeres. Incluyendo en estas cifras a los hipertensos a los que no se les detecta cifras tensionales elevadas debido a que reciben tratamiento médico, las prevalencias serían de $13,1 \%$ en hombres y $13,4 \%$ en mujeres. Para el grupo de edad MONICA (35-64 años) las cifras en hombres son $14,3 \%$ y $19,1 \%$ y $11,6 \%$ y $18,5 \%$ en mujeres, respectivamente. El grado de control de la población hipertensa calculado según la diferencia relativa de las dos tasas de prevalencia indica que sólo un $22,9 \%$ de los hombres y un $35,1 \%$ de las mujeres hipertensas de todas las edades están bajo control. Con respecto al grupo de edad MONICA, el grado de control sería similarmente de un $25,1 \%$ en los hombres y $37,3 \%$ en las mujeres.

La concentración media de colesterol sérico es de $192,5 \mathrm{mg} / \mathrm{dl}$ en hombres y 187,3 
Tabla 2

Tensión arterial media (desviación estándar) y prevalencia de hipertensión (\%) según grupos de edad y sexo: Criterio del Joint National Committee on detection, evaluation and treatment of high blood pressure, 1993

\begin{tabular}{|c|c|c|c|c|c|c|c|c|}
\hline Hombres & $\begin{array}{c}18-24 \\
n=176\end{array}$ & $\begin{array}{c}25-34 \\
n=429\end{array}$ & $\begin{array}{c}35-44 \\
n=352\end{array}$ & $\begin{array}{c}45-54 \\
n=222\end{array}$ & $\begin{array}{c}55-65 \\
n=333\end{array}$ & $\begin{array}{c}\text { Tasa } \\
\text { población } \\
18-65 \\
n=1.513\end{array}$ & $\begin{array}{c}\text { Tasa pobl. } \\
\text { estandar. } \\
18-65 \\
n=1.513\end{array}$ & $\begin{array}{c}\text { Tasa } \\
\text { truncada } \\
\text { estandar. } \\
35-64 \\
n=869\end{array}$ \\
\hline TAS media & $\begin{array}{l}125,7 \\
(12,2)\end{array}$ & $\begin{array}{r}125,7 \\
(12,7)\end{array}$ & $\begin{array}{l}128,2 \\
(14,6)\end{array}$ & $\begin{array}{l}132,9 \\
(15,8)\end{array}$ & $\begin{array}{l}142,1 \\
(19,2)\end{array}$ & $\begin{array}{l}130,8 \\
(16,3)\end{array}$ & 130,9 & 133,8 \\
\hline TAD media & $\begin{array}{r}73,0 \\
(10,9)\end{array}$ & $\begin{array}{r}74,8 \\
(10,1)\end{array}$ & $\begin{array}{r}79,5 \\
(10,9)\end{array}$ & $\begin{array}{r}81,9 \\
(9,8)\end{array}$ & $\begin{array}{r}83,8 \\
(12,2)\end{array}$ & $\begin{array}{r}78,3 \\
(11,5)\end{array}$ & 78,7 & 81,6 \\
\hline HTA definida $(\%)^{1}$ & 22,9 & 15,7 & 29,2 & 41,0 & 58,7 & 32,3 & 32,9 & 42,0 \\
\hline HTA tratada farmac. $(\%)^{2}$ & 5,4 & 4,0 & 16,0 & 17,1 & 23,7 & 16,4 & 16,1 & 18.7 \\
\hline HTA controlada $(\%)^{3}$ & 0,0 & 1,1 & 5,3 & 3,3 & 2,4 & 2,6 & 2,7 & 3,2 \\
\hline HTA tratada y controlada $(\%)^{4}$ & 0,0 & 26,9 & 33,1 & 19,5 & 10,2 & 15,6 & 16,5 & 17,3 \\
\hline HTA conocida $(\%)^{5}$ & 14,1 & 22,5 & 33,5 & 44,2 & 42,8 & 40,0 & 35,2 & 35,9 \\
\hline
\end{tabular}

\begin{tabular}{|c|c|c|c|c|c|c|c|c|}
\hline Mujeres & $\begin{array}{c}18-24 \\
n=178\end{array}$ & $\begin{array}{c}25-34 \\
n=435\end{array}$ & $\begin{array}{c}35-44 \\
n=357\end{array}$ & $\begin{array}{c}45-54 \\
n=294\end{array}$ & $\begin{array}{c}55-65 \\
n=313\end{array}$ & $\begin{array}{c}\text { Tasa } \\
\text { población } \\
18-65 \\
n=1.577\end{array}$ & $\begin{array}{c}\text { Tasa pobl. } \\
\text { estandar. } \\
18-65 \\
n=1.577\end{array}$ & $\begin{array}{c}\text { Tasa } \\
\text { truncada } \\
\text { estandar. } \\
35-64 \\
n=920\end{array}$ \\
\hline $\begin{array}{l}\text { TAS media } \\
\text { (DE) }\end{array}$ & $\begin{array}{l}114,2 \\
(11,0)\end{array}$ & $\begin{array}{l}113,8 \\
(12,5)\end{array}$ & $\begin{array}{l}118,8 \\
(15,0)\end{array}$ & $\begin{array}{l}131,0 \\
(18,1)\end{array}$ & $\begin{array}{l}141,0 \\
(21,9)\end{array}$ & $\begin{array}{l}123,3 \\
(19,3)\end{array}$ & 123,5 & $\begin{array}{l}128,6 \\
(20)\end{array}$ \\
\hline $\begin{array}{l}\text { TAD media } \\
\text { (DE) }\end{array}$ & $\begin{array}{c}68,3 \\
(9,0)\end{array}$ & $\begin{array}{l}68,9 \\
(9,9)\end{array}$ & $\begin{array}{r}74,3 \\
(10,9)\end{array}$ & $\begin{array}{r}81,2 \\
(11,3)\end{array}$ & $\begin{array}{r}83,0 \\
(12,2)\end{array}$ & $\begin{array}{r}74,7 \\
(12,2)\end{array}$ & 75,3 & $\begin{array}{r}78,8 \\
(12,0)\end{array}$ \\
\hline HTA definida $(\%)^{\mathrm{t}}$ & 4,6 & 5,0 & 11,7 & 40,7 & 60,3 & 23,7 & 23,9 & 33,8 \\
\hline HTA tratada farmac. $(\%)^{2}$ & 2,5 & 9,6 & 19,4 & 25,0 & 44,0 & 34,3 & 32,3 & 33,2 \\
\hline HTA controlada $(\%)^{3}$ & 29,3 & 9,6 & 0,6 & 10,3 & 9,4 & 9,5 & 9,0 & 8.4 \\
\hline HTA tratada y controlada $(\%)^{4}$ & 100 & 100 & 3,0 & 41,2 & 21,4 & 27,8 & 27,9 & 25,4 \\
\hline HTA conocida $(\%)^{5}$ & 59,6 & 42,7 & 38,3 & 50,3 & 62,9 & 54,9 & 56,1 & 54,6 \\
\hline
\end{tabular}

${ }^{1}$ HTA definida: TAS $\geq 140$ y $/ 6$ TAD $\geq 90$ y/ó con tratamiento farmacológico; ${ }^{2}$ HTA tratada farmacológicamente: sobre el total de hipertensos: ${ }^{3}$ HTA controlada: TAS $<140$ y TAD $<90$ (sobre el total de hipertensos); ${ }^{4}$ HTA tratada y cortrolada: TAS $<140$ y TAD $<90$ (sobre el total de hipertensos tratados farmacológicamente); ${ }^{5}$ HTA conocida: hipertensos a los que alguna vez algún medico les ha dicho que tienen la tensión arterial elevada: ${ }^{6}$ Estandarizada por la población mundial. DE: desviación estándar. 
Tabla 3

Prevalencia $(\%)$ de hipertensión observada, hipertensión real y grado de control entre la pohlación hipertensa según el criterio del estudio MONICA

\begin{tabular}{|c|c|c|c|c|c|c|c|c|}
\hline Hombres & $\begin{array}{c}18-24 \\
n=176\end{array}$ & $\begin{array}{c}25-34 \\
n=429\end{array}$ & $\begin{array}{c}35-44 \\
n=352\end{array}$ & $\begin{array}{l}45-54 \\
n=222\end{array}$ & $\begin{array}{c}55-65 \\
n=333\end{array}$ & $\begin{array}{c}\text { Tasa } \\
\text { población } \\
18-65 \\
n=1.513\end{array}$ & $\begin{array}{c}\text { Tasa pobl: } \\
\text { estandar. } \\
18-65 \\
n=1.513\end{array}$ & $\begin{array}{c}\text { Tasa } \\
\text { trunccida } \\
\text { estandar. } \\
35-64 \\
n=869\end{array}$ \\
\hline HTA observada ${ }^{\prime}$ & 4,6 & 1,6 & 8,2 & 11,3 & 26,1 & 10,1 & 10,1 & 14,3 \\
\hline HTA real $^{2}$ & 4,6 & 2,2 & 11,7 & 15,1 & 33,6 & 13,1 & 13.2 & 19.1 \\
\hline HTA controlada ${ }^{3}$ & 0 & 27,3 & 30,0 & 25,2 & 22,3 & 22,9 & 23.5 & 25, \\
\hline
\end{tabular}

\begin{tabular}{|c|c|c|c|c|c|c|c|c|}
\hline Mujeres & $\begin{array}{c}18-24 \\
n=178\end{array}$ & $\begin{array}{c}25-34 \\
n=435\end{array}$ & $\begin{array}{c}35-44 \\
n=357\end{array}$ & $\begin{array}{c}45-54 \\
n=294\end{array}$ & $\begin{array}{c}55-65 \\
n=313\end{array}$ & $\begin{array}{c}\text { Tasa } \\
\text { población } \\
\text { I8-65 } \\
n=1.577\end{array}$ & $\begin{array}{c}\text { Tasa pobl. } \\
\text { estandar. } \\
18-65 \\
n=1.577\end{array}$ & $\begin{array}{c}\text { Tasa } \\
\text { trancadla } \\
\text { estandar. } \\
35-64 \\
n=920\end{array}$ \\
\hline HTA observada' & 0,2 & 0,9 & 3,6 & 11,4 & 27,6 & 8,7 & 8,3 & 11,6 \\
\hline HTA real $^{2}$ & 1,5 & 1,3 & 4,6 & 19,1 & 41,1 & 13,4 & 12,9 & 18,5 \\
\hline HTA controlada $^{3}$ & 86,7 & 30,8 & 21,7 & 40,3 & 32,8 & 35,1 & 35,7 & 37,3 \\
\hline
\end{tabular}

${ }^{3}$ HTA observada: TAS $\geq 160 \mathrm{y} / 0 \mathrm{TAD} \geq 95 ;{ }^{2}$ HTA real: TAS $\geq 160 \mathrm{y} / 0 \mathrm{TAD} \geq 95$ y/o con tratamiento farnacológico; ${ }^{3}$ HTA controlada: HTA real (\%) -HTA observada (\%)/HTA real (\%): estandarizada por la población mundial.

$\mathrm{mg} / \mathrm{dl}$ en mujeres (tabla 4) observándose un patrón similar al descrito para la TAS y TAD. Mientras en los grupos de edades mas jóvenes los hombres tienen concentraciones de colesterol sérico más elevadas, en el grupo de edad más avanzado las concentraciones medias en mujeres superan las de los hombres. Las cifras medias de la fracción HDL del colesterol son más elevadas en las mujeres $(67,1 \mathrm{mg} / \mathrm{dl})$ que en hombres $(57,6$ $\mathrm{mg} / \mathrm{dl}$ ) manteniéndose más elevadas en éstas para todos los grupos de edad y sin un claro patrón descendente al aumentar ésta.

Por último, las concentraciones medias de trigliceridos son más elevadas en los hombres $(136,1 \mathrm{mg} / \mathrm{dl})$ que en las mujeres $(96,9 \mathrm{mg} / \mathrm{dl})$ para todas las edades y con un claro patrón ascendente al incrementarse esta.

I a tabla 5 indica que, globalmente, a un $6,7 \%$ de los hombres y $8,2 \%$ de las mujeres de la Región de Murcia alguna vez un mé- dico le ha dicho que tiene la glucosa sérica elevada o que padecían diabetes. Aproximadamente un $3,2 \%$ de hombres y un $3,6 \%$ de mujeres, además de haber recibido esta información por parte de su medico, están realizando algún tipo de tratamiento, bien dietético o farmacológico. El 11,2\% de los diabéticos y el 23,5\% de las diabéticas están siendo tratadas con insulina, mientras el $50,2 \%$ de los hombres y el $39,8 \%$ de las mujeres toman antidiabéticos orales. Casi un 40\% de las personas diabéticas están tratadas tan sólo con dieta. El Índice de Masa Corporal medio en hombres es igual al de las mujeres $(26,7)$ aunque con mayor variabilidad en éstas, como lo demuestra la desviación estándar más amplia. Sin embargo, por grupos de edad existe un patrón diferente. En los grupos de edad más jóvenes los hombres sobrepasan a las mujeres, mientras a partir de los 35-44 años, las mujeres presentan un IMC más elevado siendo esta diferencia sobre todo evidente en las mujeres de mayor edad (tabla 5). 
Tabla 4

Colesterol total, Colesterol-HDL y trigliceridos según grupos de edad y sexo: media (desviación estándar) en mg/100 ml.

\begin{tabular}{|c|c|c|c|c|c|c|c|c|}
\hline Hombres & $\begin{array}{c}18-24 \\
n=112\end{array}$ & $\begin{array}{c}25 \cdot 34 \\
n=299\end{array}$ & $\begin{array}{c}35-44 \\
n=266\end{array}$ & $\begin{array}{c}45-54 \\
n=162\end{array}$ & $\begin{array}{c}55-65 \\
n=237\end{array}$ & $\begin{array}{c}\text { Tasa } \\
\text { población } \\
18-65 \\
n=1.076\end{array}$ & $\begin{array}{c}\text { Tasa pobl. } \\
\text { estandar. } \\
18-65 \\
n=1.076\end{array}$ & $\begin{array}{c}\text { Tasa } \\
\text { trumcada } \\
\text { estándar. } \\
35-64 \\
n=6.34\end{array}$ \\
\hline Colesterol total & $\begin{array}{l}165,3 \\
(37,4)\end{array}$ & $\begin{array}{l}184,5 \\
(42,2)\end{array}$ & $\begin{array}{l}200,6 \\
(47,6)\end{array}$ & $\begin{array}{l}203,9 \\
(47,6)\end{array}$ & $\begin{array}{l}210,3 \\
(49,0)\end{array}$ & $\begin{array}{l}192,5 \\
(47,2)\end{array}$ & 194,7 & 206,7 \\
\hline Colesterol HDL ${ }^{1}$ & $\begin{array}{r}58,3 \\
(15,5)\end{array}$ & $\begin{array}{r}56,9 \\
(16,0)\end{array}$ & $\begin{array}{r}57,1 \\
(17,8)\end{array}$ & $\begin{array}{r}59.7 \\
(18,9)\end{array}$ & $\begin{array}{r}57,0 \\
(16,8)\end{array}$ & $\begin{array}{r}57,6 \\
(17,1)\end{array}$ & 57,5 & 57,8 \\
\hline Triglicéridos $^{2}$ & $\begin{array}{l}110,6 \\
(63.4)\end{array}$ & $\begin{array}{l}128,1 \\
(72,9)\end{array}$ & $\begin{array}{c}145,4 \\
(103,4)\end{array}$ & $\begin{array}{r}153,0 \\
(87.9)\end{array}$ & $\begin{array}{l}148.7 \\
(94.9)\end{array}$ & $\begin{array}{l}136,1 \\
(88.7)\end{array}$ & 139.1 & 149.2 \\
\hline
\end{tabular}

\begin{tabular}{|c|c|c|c|c|c|c|c|c|}
\hline Mujeres & $\begin{array}{c}18-24 \\
n=124\end{array}$ & $\begin{array}{c}25-34 \\
n=331\end{array}$ & $\begin{array}{c}35-44 \\
n=279\end{array}$ & $\begin{array}{c}45-54 \\
n=226\end{array}$ & $\begin{array}{c}55-65 \\
n=245\end{array}$ & $\begin{array}{c}\text { Tasa } \\
\text { poblacion } \\
18-65 \\
n=1.205\end{array}$ & $\begin{array}{c}\text { Tasa pobl. } \\
\text { estandar. } \\
18-65 \\
n=1.205\end{array}$ & $\begin{array}{c}\text { Total } \\
\text { truncada } \\
\text { estädar. } \\
35-64 \\
n=714\end{array}$ \\
\hline Colesterol total & $\begin{array}{l}160,8 \\
(34,4)\end{array}$ & $\begin{array}{l}174,3 \\
(36,4)\end{array}$ & $\begin{array}{l}187,1 \\
(43,3)\end{array}$ & $\begin{array}{l}200,4 \\
(41,7)\end{array}$ & $\begin{array}{l}215,1 \\
(46,9)\end{array}$ & $\begin{array}{l}187,3 \\
(43,8)\end{array}$ & 188,4 & 201,3 \\
\hline Colesterol HDL ${ }^{\prime}$ & $\begin{array}{r}72,2 \\
(19,7)\end{array}$ & $\begin{array}{r}64,6 \\
(18,3)\end{array}$ & $\begin{array}{r}66,6 \\
(17,5)\end{array}$ & $\begin{array}{r}66,4 \\
(19,6)\end{array}$ & $\begin{array}{r}67,5 \\
(18,0)\end{array}$ & $\begin{array}{r}67,1 \\
(18,5)\end{array}$ & 66,7 & 66,6 \\
\hline Triglicéridlos & $\begin{array}{r}77,3 \\
(38,6)\end{array}$ & $\begin{array}{r}81,4 \\
(36,9)\end{array}$ & $\begin{array}{r}92,1 \\
(45,0)\end{array}$ & $\begin{array}{l}110,6 \\
(60,2)\end{array}$ & $\begin{array}{l}126,4 \\
(65,5)\end{array}$ & $\begin{array}{r}96,9 \\
(52,9)\end{array}$ & 97,8 & 107.1 \\
\hline
\end{tabular}

En 1.070 hombres y 1.200 mujeres; ${ }^{2}$ En 1.075 hombres; ${ }^{3}$ Estandarizada por la población mundial.

La distribución en percentiles de las kilocalorías/día de gasto energético en el tiempo libre en la población, se puede observar en las figuras 1 y 2 . La actividad física, en los hombres, es mayor en las edades más jóvenes y más avanzadas que en los grupos de edades medias. Sin embargo, en todos los grupos de edad existe un amplio rango de variabilidad, indicando la existencia de hombres físicamente activos en cada uno de ellos. En las mujeres, el consumo energético por actividad física de tiempo libre es únicamente existente en las edades más jóvenes, para disminuir y mantenerse en niveles bajos y estables en los demás grupos de edad.

La figura 3 presenta la distribución de la muestra según el número de factores de riesgo presentes considerando tan sólo el tabaco, el colesterol elevado ( $\geq 200 \mathrm{mg} / \mathrm{dl}$ ) y la hipertensión (TAS $\geq 140$ y/o $\mathrm{TAS} \geq 90 \mathrm{y} / \mathrm{o}$ con tratamiento farmacológico). Globalmente, el $84,7 \%$ de los hombres y el $71,7 \%$ de las mujeres participantes presentaron al menos uno de estos factores. Aproximadamente el $40,5 \%$ de los hombres y la mitad de esta cifra en mujeres $(20,9 \%)$ presentaron dos o más factores de riesgo asociados. Si consideramos los factores de riesgo aisladamente (figura 4) el consumo habitual de cigarrillos es el más frecuente. La combinación de factores de riesgo más frecuente en los hombres es la del tabaco junto a la hipercolesterolemia $(15,3 \%)$. En las mujeres, la hipertension y la hipercolesterolemia $(12,4 \%)$. Un $8,5 \%$ de los hombres y un $0,8 \%$ de las mujeres presentaron los tres factores de riesgo simultáneamente.

\section{DISCUSIÓN}

Este estudio presenta la prevalencia de los principales factores de riesgo cardiovascular 
Tabla 5

Prevalencia (\%) de elevación de glucosa sérica y diabetes declarada: tratamiento farmacológico y dietético. Índice de Masa Corporal (IMC) según sexo: media (desviación estándar)

\begin{tabular}{|c|c|c|c|c|c|c|c|c|}
\hline Hombres & $\begin{array}{c}18-24 \\
n=177\end{array}$ & $\begin{array}{c}25-34 \\
n=429\end{array}$ & $\begin{array}{c}35-44 \\
n=353\end{array}$ & $\begin{array}{c}45-54 \\
n=222\end{array}$ & $\begin{array}{c}55-65 \\
n=333\end{array}$ & $\begin{array}{c}\text { Tasa } \\
\text { población } \\
18-65 \\
n=1.514\end{array}$ & $\begin{array}{c}\text { Tasa } \\
\text { pobl. } \\
\text { estandar. } \\
18-65 \\
n=1.514\end{array}$ & $\begin{array}{c}\text { Tasa } \\
\text { truncada } \\
\text { estandar. } \\
35-64 \\
n=869\end{array}$ \\
\hline $\begin{array}{l}\text { Alguna vez elevación } \\
\text { glucosa sérica }(\%)^{\text {l }}\end{array}$ & 2,9 & 2,0 & 5,4 & 8,1 & 16,0 & 6,7 & 6,7 & 8,8 \\
\hline Diabetes $(\%)$ declarada ${ }^{2}$ & 1,5 & 0,05 & 1,9 & 3.7 & 9,3 & 3.2 & 3,1 & 4,2 \\
\hline $\begin{array}{l}\text { Diabetes tratada con } \\
\text { insulina }(\%)\end{array}$ & 0 & 0 & 0 & 0 & 16,9 & 11,2 & 9.6 & 7,5 \\
\hline $\begin{array}{l}\text { Diabetes tratada con } \\
\text { antidiabéticos orales }(\%)\end{array}$ & 0 & 0 & 47,1 & 65,1 & 53,8 & 50.2 & 51,4 & 58,0 \\
\hline $\begin{array}{l}\text { Diabetes tratada sólo } \\
\text { con dieta }(\%)\end{array}$ & 100 & 100 & 52,7 & 35,0 & 29,3 & 38,7 & 40 & 34,5 \\
\hline IMC & $\begin{array}{l}25,9 \\
(3,2)\end{array}$ & $\begin{array}{r}25,9 \\
(3,6)\end{array}$ & $\begin{array}{l}26,6 \\
(3,2)\end{array}$ & $\begin{array}{l}28,1 \\
(3,7)\end{array}$ & $\begin{array}{l}27,8 \\
(3,6)\end{array}$ & $\begin{array}{l}26,7 \\
(3,6)\end{array}$ & 26,9 & 27,4 \\
\hline
\end{tabular}

\begin{tabular}{|c|c|c|c|c|c|c|c|c|}
\hline Mujeres & $\begin{array}{c}18-24 \\
n=178\end{array}$ & $\begin{array}{c}25-34 \\
n=435\end{array}$ & $\begin{array}{c}35-44 \\
n=357\end{array}$ & $\begin{array}{c}45-54 \\
n=294\end{array}$ & $\begin{array}{c}55-65 \\
n=313\end{array}$ & $\begin{array}{c}\text { Tasa } \\
\text { población } \\
18-65 \\
n=1.577\end{array}$ & $\begin{array}{c}\text { Tasa pobl. } \\
\text { estandar. } \\
18-65 \\
n=1.577\end{array}$ & $\begin{array}{c}\text { Tasa } \\
\text { truncada } \\
\text { estandar. } \\
35-64 \\
n=920\end{array}$ \\
\hline $\begin{array}{l}\text { Alguna vez elevación } \\
\text { glucosa sérica }(\%)^{\prime}\end{array}$ & 1,2 & 2,3 & 6,0 & 8,8 & 22,5 & 8,2 & 7,6 & 9,8 \\
\hline Diabetes $(\%)$ declarada ${ }^{2}$ & 0,2 & 0,3 & 0,8 & 4,3 & 12,4 & 3,6 & 3,1 & 4,3 \\
\hline $\begin{array}{l}\text { Diabetes tratada con } \\
\text { insulina }(\%)\end{array}$ & 100 & 27,6 & 0 & 34,3 & 21,2 & 23,5 & 19,2 & 20,9 \\
\hline $\begin{array}{l}\text { Diabetes tratada con } \\
\text { antidiabéticos orales }(\%)\end{array}$ & 0 & 0 & 26,0 & 37,0 & 42,9 & 39,8 & 41,8 & 40,5 \\
\hline $\begin{array}{l}\text { Diabetes tratada sólo } \\
\text { con dieta }(\%)\end{array}$ & 0 & 72,4 & 74 & 28,7 & 35,9 & 36,8 & 40,0 & 38,6 \\
\hline IMC & $\begin{array}{l}22,9 \\
(3,4)\end{array}$ & $\begin{array}{l}24,2 \\
(3,8)\end{array}$ & $\begin{array}{l}26,8 \\
(4,5)\end{array}$ & $\begin{array}{l}29,7 \\
(4,6)\end{array}$ & $\begin{array}{l}30,8 \\
(4,7)\end{array}$ & $\begin{array}{l}26,7 \\
(5,0)\end{array}$ & 26,9 & 28,5 \\
\hline
\end{tabular}

'Alguna vez un médico le ha dicho que tiene la glucisa alta en la sangre, que tiene azúcar en la sangre o que es diabético, ${ }^{2}$ Alguna vez un médico le ha dicho que tiene la glucosa alta en la sangre y, durarte las dos últimas semanas, está tomando medicación o está hacienuio algún régimen para disminuir el azúcar en la sangre: ${ }^{3}$ Estandarizada por la población mundial. 
Figura 1

Distribución (\%) kilocalorías/día de gasto energético: Actividad física de tiempo libre en percentiles. Hombres

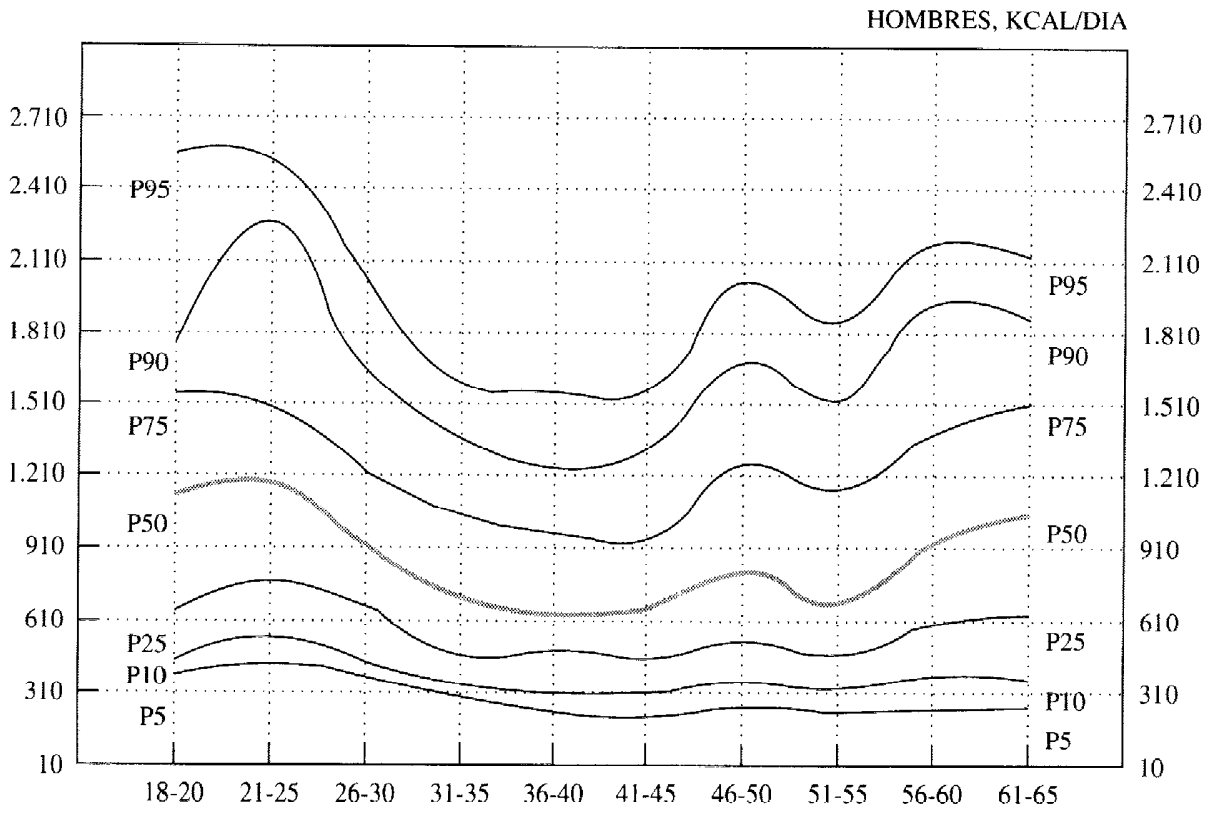

GRUPOS DE EDAD EN AÑOS

Figura 2

Distribucion (\%) kilocalorías/día de gasto energético: Actividad física de tiempo libre en percentiles. Mujeres

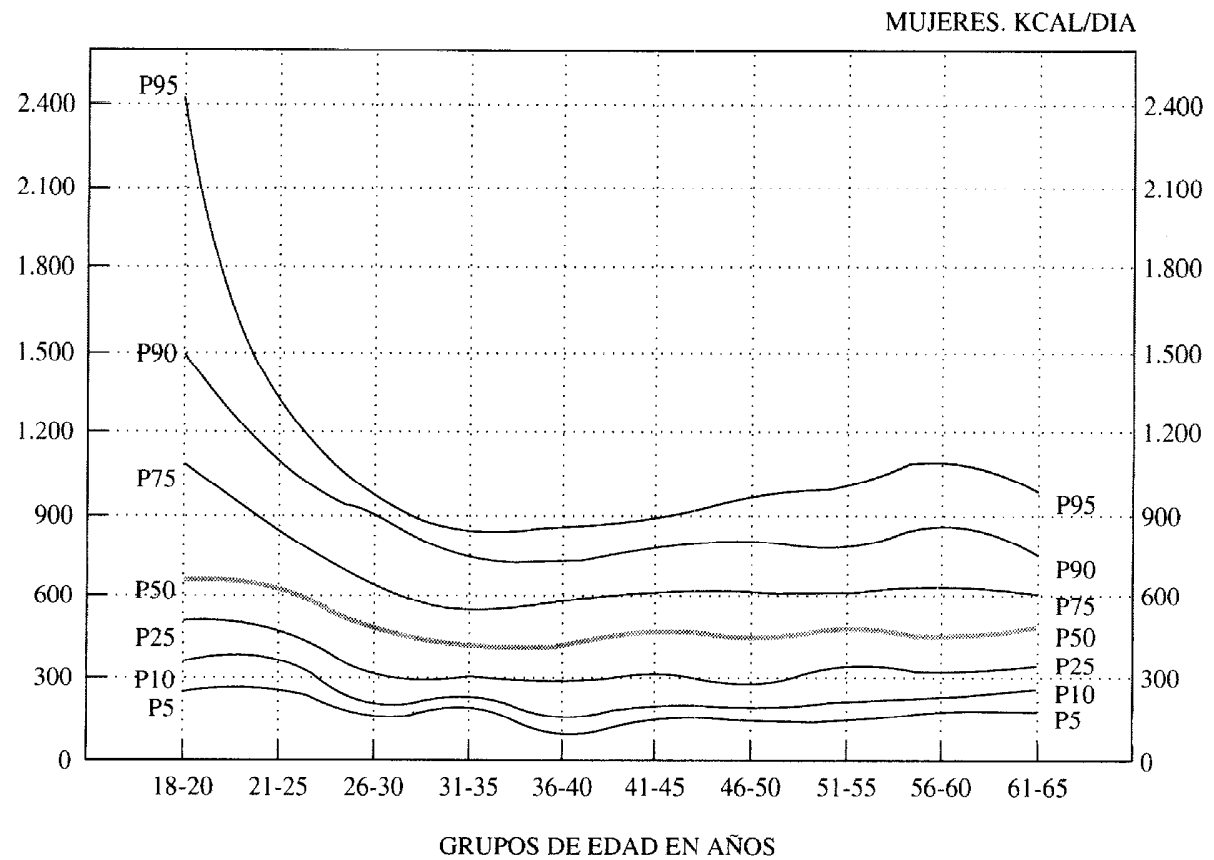


Figura 3

Distribución (\%) de pohi i ion ie $18-65$ años según el número de factores de riesgo cardiovascular por sexo

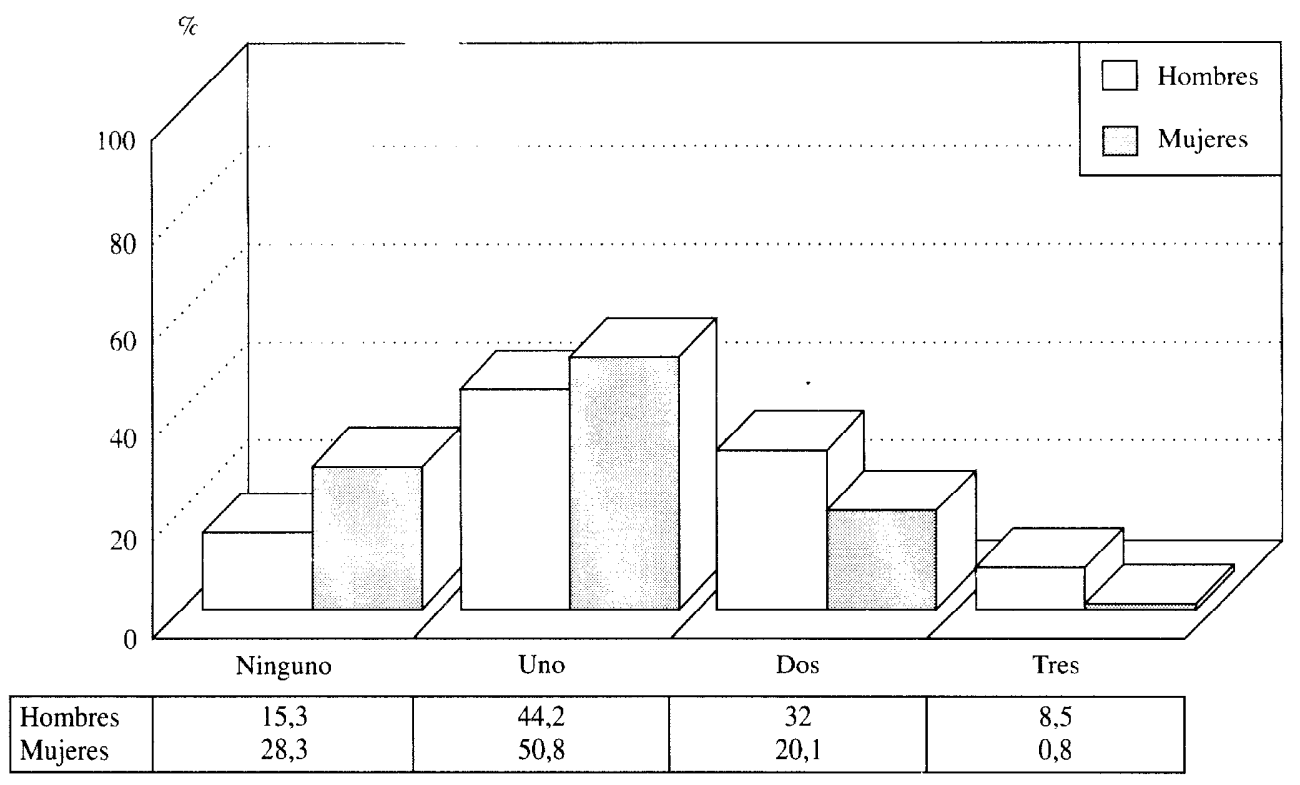

FACTORES DE RIESGO

Figura 4

Distribución (\%) de población de 18-65 años según combinaciones de los principales factores de riesgo cardiovascular por sexo

FACTORES DE RIESGO

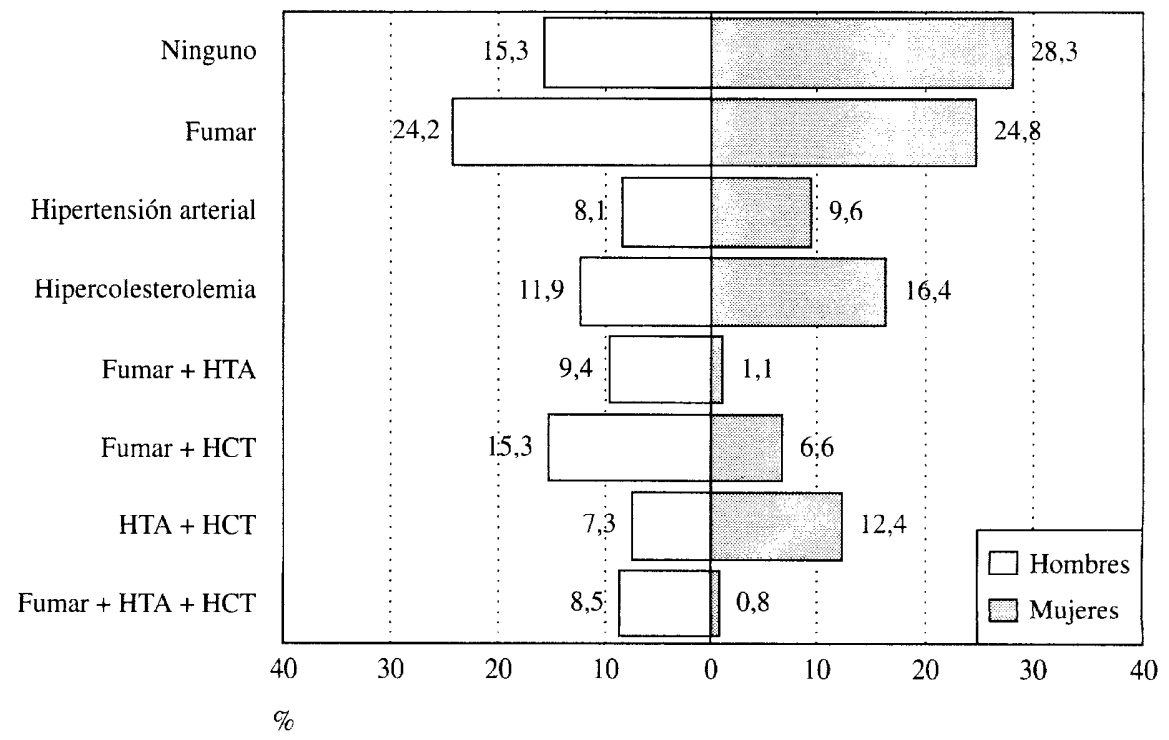


en un área de España de alta mortalidad coronaria y cerebrovascular. La principal limitación a la hora de interpretar los resultados es la baja tasa de respuesta lograda. Para intentar conocer algunas de las características de la población que no respondió, se realizó una encuesta telefónica a una muestra de no respuestas. La principal diferencia entre respuestas y no respuestas fue la menor prevalencia de hipertensión en éste último grupo. Así, las cifras de hipertensión presentadas en este trabajo pueden estar ligeramente sobrestimadas. Sin embargo, la ponderación de las tasas brutas por el inverso de la tasa de respuesta penaliza los grupos con una mayor tasa de respuesta, que son al mismo tiempo los de mayor edad y mayor prevalencia de HTA. Por tanto, parte de esta sobrestimación se ha corregido a nivel de cada estrato muestral (edad, sexo, tipo de residencia y área sanitaria). Aún así, el efecto de la baja tasa de respuesta sobre la prevalencia estimada de lípidos plasmáticos es difícil de valorar, aunque la elevación plasmática de estos suele ocurrir de forma más silente e inadvertida por los sujetos que la elevación de la tensión arterial, siendo el sesgo de selección esperable de menor magnitud.

Para poner en contexto la importancia de las cifras es necesario compararlas con estudios similares. La tabla 6 presenta los datos comparativos de nuestro estudio con un estudio similar llevado a cabo en 1989 sobre una muestra aleatoria y representativa de la población adulta española de 35 a 64 años ${ }^{15}$ y algunos datos publicados por el centro MONICA-Cataluña" ${ }^{16}$, todos ellos realizados con metodologías similares. De este último estudio existen dos trabajos publicados. uno con una muestra mayor ${ }^{16}$ que el otro". Siempre que ha sido posible (en este caso, todas las variables excepto el porcentaje de hipertensión, de hipertensión real y de grado

Tabla 6

Comparación de resultados: Estudio de factores de riesgo cardiovascular de la Región de Murcia Estudio en pabbación adulta españoh de 35 a 64 años y Estudio MONICA-Cataluña

\begin{tabular}{|c|c|c|c|c|c|c|c|c|c|c|}
\hline & $\begin{array}{c}\text { Tamaño } \\
\text { muestral }\end{array}$ & $\begin{array}{c}\text { Fumudores } \\
\text { habituales } \\
(\%)\end{array}$ & $\begin{array}{c}\text { Ex- } \\
\text { fumadores } \\
(k)\end{array}$ & $\begin{array}{c}\text { TAS } \\
\text { minHg: } \\
\text { media } \\
(D E)^{1}\end{array}$ & $\begin{array}{c}\text { TAD } \\
\text { munHg: } \\
\text { mediug } \\
(D E)^{1}\end{array}$ & $\begin{array}{l}\text { Hiper. } \\
\text { rension } \\
(G)^{\prime}\end{array}$ & $\begin{array}{c}\text { Hiperten } \\
\text { sion } \\
\text { real } \\
(\$)^{2}\end{array}$ & $\begin{array}{l}\text { Gredo } \\
\text { comatrol } \\
\text { hiprestens } \\
\text { as tht }\end{array}$ & $\begin{array}{c}\text { Comesiern } \\
\text { fmghtll: } \\
\text { media (DE) }\end{array}$ & $\begin{array}{l}\text { MC: } \\
\text { media } \\
\text { OEE }\end{array}$ \\
\hline \multicolumn{11}{|l|}{ HOMBRES } \\
\hline Murcia. 1992 & 1.514 & 49,9 & 15.7 & 133,8 & 81,6 & 14.3 & 19.1 & 25.1 & $\begin{array}{l}206.4 \\
(47.81\end{array}$ & $\begin{array}{l}27,4 \\
(3,5)\end{array}$ \\
\hline España, 1989 & 810 & 49,4 & 24,9 & $\begin{array}{r}13,4,4^{!} \\
(19,5) \\
\end{array}$ & $\begin{array}{r}84,5^{1} \\
(11,91 \\
\end{array}$ & $21,6^{i}$ & $29.3^{2}$ & 26.7 & $\begin{array}{l}210.1 \\
(440)\end{array}$ & $\begin{array}{r}27.2 \\
(3.4)\end{array}$ \\
\hline $\begin{array}{l}\text { MONICA- } \\
\text { Cataluna, 1986-8 }\end{array}$ & 993 & 46,6 & 23,2 & $\begin{array}{r}123,4^{1} \\
(17.2) \\
\end{array}$ & $\begin{array}{r}74,9^{1} \\
(10.6) \\
\end{array}$ & $6.3^{1}$ & 8.4 & 25 & $\begin{array}{r}224.5 \\
(41,6)\end{array}$ & $\begin{array}{r}26.1 \\
(3.2) \\
\end{array}$ \\
\hline \multicolumn{11}{|l|}{ MUJERES } \\
\hline Murcia, 1992 & 1.577 & 15,9 & 3,1 & 128,6 & 78,8 & 11.6 & 18.5 & 37,3 & $\begin{array}{r}201.3 \\
(45.0) \\
\end{array}$ & $\begin{array}{r}28.5 \\
(4.8) \\
\end{array}$ \\
\hline España, 1989 & 1.211 & 16,7 & 5,0 & $\begin{array}{r}131,6^{1} \\
(20,9)\end{array}$ & $\begin{array}{r}83,1^{1} \\
(12,0) \\
\end{array}$ & $18.1^{i}$ & $26.6^{2}$ & 32 & $\begin{array}{l}211.9 \\
(42.2) \\
\end{array}$ & $\begin{array}{r}27.6 \\
(4,8) \\
\end{array}$ \\
\hline $\begin{array}{l}\text { MONICA- } \\
\text { Cataluña, 1986-8 }\end{array}$ & 994 & 6,7 & 2,2 & $\begin{array}{l}121,5^{1} \\
(18,3)\end{array}$ & $\begin{array}{r}73,3^{!} \\
(10,6)\end{array}$ & $3,6^{1}$ & 13 & 72.3 & $\begin{array}{l}220,6 \\
(41,6)\end{array}$ & $\begin{array}{l}27.3 \\
(4.5)\end{array}$ \\
\hline
\end{tabular}

DE: desviación estándar: ' Estos estudios se han realizado con esfigmomanómetros de cero aleatorio; ${ }^{2}$ se incluyen también a las personas con tratamiento dietético exclusivamente. 
de control) se ha incluido la información del estudio con mayor muestra. En cuanto a las prevalencias de fumadores habituales son más bajas en ambos sexos en el centro MONICA-Cataluña y, similares en los otros dos estudios. En cambio, las cifras de ex-fumadores son particularmente bajas en el estudio de Murcia. Las tensiones medias sistólicas y diastólicas son consistentemente más bajas en el centro MONICA-Cataluña. Esto se traduce en unas cifras de hipertensión, tanto observada como real, entre 3-4 veces más bajas en este centro. Existen también discrepancias entre el estudio de Murcia y el de la población adulta española, en el sentido de una mayor hipertensión en este último. Parte de las discrepancias observadas se pueden deber a los diferentes aparatos de medida utilizadas. El estudio de Murcia se realizó con esfigmomanómetro de mercurio aneroide normal y los otros dos con modelos especiales de cero aleatorio. Se han descrito diferencias sistemáticas ${ }^{17,18}$ en la utilización de estos últimos, en el sentido de subestimar las cifras tensionales, particularmente las diastólicas. Por tanto, tendrían sentido las bajas cifras de hipertensión calculadas en el centro MONICA. En cuanto a las elevadas prevalencias encontradas en el estudio de la población adulta española, en parte se pueden deber, en el caso de la hipertensión real, a la inclusión como hipertensas a aquellas personas que declaran seguir tratamiento dietético, cuando los otros dos estudios incluyen tan sólo los tratamientos farmacológicos.

El porcentaje de control es muy similar, sin embargo, entre centros, excepción hecha para las mujeres del centro MONICA. Esta disparidad puede deberse a la pequeña muestra sobre la que están calculadas estas cifras (389 mujeres en total) y ser fruto de variabilidad aleatoria. Globalmente puede decirse que una cuarta parte de los hombres y una tercera parte de las mujeres que sufren hipertensión la tienen bajo control.

Las cifras promedio de colesterol sérico son consistentemente más elevadas en el centro MONICA y menores en el estudio de Murcia. Contrariamente, el IMC es substancialmente más elevado en las mujeres del estudio de Murcia.

En resumen, excepto para la mayor hipertensión detectada en el conjunto de España, el estudio de la región de Murcia muestra patrones similares a éste. Los resultados del centro MONICA destacan sobre el conjunto en cuanto a una menor hipertensión y una mayor concentración sérica de colesterol.

En el contexto internacional, comparando los datos para la población de 35-64 años de este área del sur del mediterráneo que es la Región de Murcia (ver tabla 6) con los datos ofrecidos por los 40 centros del estudio MO$\mathrm{NICA}^{11}$, a los que se ha ordenado de menor a mayor frecuencia de los factores de riesgo y de los que se ofrece el valor mínimo/máximo para hombres y mujeres respectivamente, se puede decir que la Región de Murcia está situada en el extremo inferior de la distribución para la hipertensión real (hombres: $8,4 \%$ Cataluña/45,3\% Kuopio, Finlandia; mujeres: $12,6 \%$ Glostrup, Dinamarca/40,5\% Ex-Republica Democrática Alemana) y en el extremo medio-alto en cuanto al grado de control de ésta (hombres: $5 \%$ Varsovia/42\% Rhein, República Federal Alemana; mujeres: $10 \%$ Varsovia/73\% Gante, Bélgica). En el consumo de tabaco, los hombres se situarían en el extremo más elevado de la distribución $(23,7 \%$ Suecia/58,8\% Novosibirsk, Rusia) mientras que ambos sexos se situarían en el extremo más bajo de la distribución en cuanto a colesterol sérico (hombres: 158,7 mg/dl Beijing, China/247,7 mg/dl Luxemburgo; mujeres: 162,5 $\mathrm{mg} / \mathrm{dl}$ Beijing, China/247,7 mg/dl Glasgow). En la distribución de países con estos tres factores de riesgo simultáneamente, las mujeres de Murcia estarían en el extremo más bajo de la distribución con una prevalencia de $0,2 \%(0,1 \%$ Beijing, China $/ 5,4 \%$ Glasgow), en cambio los hombres estarian situados en posición intermedia con una prevalencia del 2,7\% (0,3\% Beijing, China/9,1\% Kuopio, Finlandia) debido al elevado consu- 
mo de tabaco. Por último, ambos sexos se encuentran en el extremo más elevado de IMC medio (hombres: 23,4 Beijing/27,5 Kaunas, Lituania; mujeres: 23,5 Glostrup/ 29,3 Kaunas). Esta elevada presencia de obesidad es consistente con la descrita en la limítrofe Comunidad Valenciana ${ }^{19}$. Además, su mayor impacto cn las mujeres podría estar en parte explicado por el diferente consumo energético debido a actividad física, ya que mientras los hombres se mantienen con un cierto nivel de actividad tanto en los grupos de edad más jóvenes que en los más avanzados, las mujeres prácticamente abandonan la actividad física al alcanzar la década de los 30 años.

Comparativamente con los datos de estudios anteriores realizados en la Región de Murcia es importante señalar que mientras la prevalencia global de hipertensión parece haber sufrido ligeras modificaciones, el grado de control de los hipertensos ha mejorado sensiblemente pasando del $5 \%$ al $30 \%$ en la actualidad $^{5}$. Parte del descenso en la mortalidad cerebrovascular observada en los últimos 17 años ${ }^{2,3}$ puede deberse a este excelente indicador. Por otra parte, los únicos dalos adicionales de los que se tiene información de años anteriores al de la encuesta es el consumo de tabaco: el consumo de tabaco era ya elevado en los datos obtenidos por la Encuesta Nacional de Salud en $1987^{\circ}$ y sigue siendo elevado en la ulterior encuesta realizada en $1993^{20}$, donde además se ponía en evidencia que la Región de Murcia era una de las pocas áreas de España sin tendencia decreciente en el consumo de tabaco.

Globalmente, la situación de la Región de Murcia no parece tan desfavorable en cuanto a la presencia de factores de riesgo cardiovascular, a excepción del exceso en el consumo de tabaco y en la obesidad, sobre todo en las mujeres. Aunque ambos son importantes factores independientes de cardiopatía coronaria ${ }^{21.22}$ parece que deban existir factores asociados al manejo del paciente coronario (acceso las unidades coronarias, diagnostico precoz, etc.) que puedan expli- car la desfavorables situación que en cuanto a la mortalidad coronaria tiene la Region de Murcia en el contexto español. Habrá que esperar a los resultados del proyecto IBERI$\mathrm{CA}^{2.4}$ (red de registro de infartos de miocardio en ocho CC.AA.) para conocer qué parte de esta situación desfavorable se debe a factores de riesgo ligados a la asistencia y terapéutica del paciente coronario.

\section{AGRADECIMIENTOS}

Los autores desean agradecer a Iñaki Moreno Sueskun la ayuda en el diseño del cuestionario de actividad física, a Ángeles Aragón Martínez el trabajo realizado con la extracción y procesamiento de muestras, a Mukund Nargundkar (Special Surveys Division Statistics, Health \& Welfare, Ottawa, Canadá) la ayuda técnica en aspectos de recomposición de la muestra, a Marc Saéz (Universitat POMPEU FABRA) la colaboración en el cálculo de los coeficientes de variación y a los asesores técnicos (Dr. Abellán Alemán J, Antón Botella JJ, Gomis Cebrián R, Guillen Pérez JJ, Hernando Arizaleta L, Martínez Hernández P, Párraga Sánchez B, Sánchez de Toro Larios JM, Tébar Massó FJ, Villegas García M, Gómez Gerique JA, Stachenko SJ, Petrasovits A, Ayuso Giner E, Rodríguez Artalejo F, Banegas Banegas JR, Cirera Suárez Ll) por la ayuda prestada en diversos aspectos del trabajo.

\section{BIBLIOGRAFÍA}

1. Segura A, Olivares O, Oñorbe JA, Mata M. Evolución de las diferencias en salud en los últimos años por Comunidades Autónomas. En: Navarro C, Cabasés J, Tormo MJ, eds. Informe SESPAS 1995: La Salud y el Sistema Sanitario en España. Barcelona: SG Editores; 1995. p.3-17.

2. Rodríguez P, Navarro C y Tormo MJ. Mortalidad cardiovascular en Murcia 1975-1982. Análisis de tendencias. Rev Esp Cardiol 1989; 42 (Supl 1): 62-66.

3. Cirera Ll, Tormo MJ, Navarro C. Evolución de la mortalidad cardiovascular, isquémica y cerebrovascular en la Región de Murcia. Período, 197591. Rev Lat Cardiol 1996; 17:39-43. 
4. Ramírez Romero P, Moreno Guillen S, Abellán Alemán J, Merino Gálvez E, Mcrino Sánchez J. Prevalencia de la hipertensión arterial y su distribución en la población murciana. Med Clin (Barc) 1984; 83:188-191.

5. Moreno Guillén S, Ramírez Moreno P, Abellán Alemán J, Merino Sánchez J. El diagnóstico, la educación sanitaria y el grado de control de los hipertensos adultos de la región de Murcia. Med Clin (Barc) 1984; 83:101-5.

6. Ministerio de Sanidad y Consumo. Encuesta Nacional de Salud 1987. Madrid: Ministerio de Sanidad y Consumo; 1989.

7. WHO MONICA Project. Part III: Population Survey; Section 1: Population survey data component. In: MONICA Manual. Geneva, 1990.

8. Tormo MJ, Navarro C, Pérez-Flores D. Quality control in the measurement of blood pressure: a practical estimation of within-observer variability in community surveys. Eur J Publ Health 1995; 5: $265-8$.

9. The fith report of the Joint National Committee on Detection, Evaluation and Treatment of high blood pressure. Arch Intern Med 1993; 153:15483.

10. WHO. Arterial Hypertension. Report of a WHO Expert Committee. Geneve: WHO Technical Report Series 628, 1978.

11. WHO MONICA Project. WHO MONICA Project: Risk Factors. Int J Epidemiol 1989 (supl 1): S46-S55.

12. Tormo MJ, Moreno I, Chirlaque MD, Navarro C. Validez de un cuestionario de actividad física reciente. Gac San 1995; 9:174-82.

13. Ministerio Sanidad y Consumo. Consenso para el control de la colesterolemia en España. Madrid: Ministerio de Sanidad y Consumo; 1989.
14. Kalton G. Introduction to Survey Sampling. Newbury Park, California: Sage University Papers, Series: Quantitative Applications in the Social Sciences, 1983: 69-75.

15. Banegas Banegas JR, Villar Álvarez F, Pérez de Andrés C, Jiménez García-Pascual R, Gil López E, Muñiz Garcia J y Juane Sánchez R. Estudio epidemiológico de los factores de riesgo cardiovascular en la población española de 35 a 64 años. Rev San Hig Púb 1993; 67:419-45

16. WHO. The WHO MONICA Project: a worldwide monitoring system for cardiovascular diseases. World Health Stat Annu 1989; 27-148.

17. Silman AJ. Failure of random zero sphygmomanometer in general practice. BMJ 1985; 290:1781-5.

18. O'Brien E, Mee F, Atkins N, O'Malley. Inaccuracy of the Hawksley random zero sphygmomanometer. Lancet 1990; 336:1465-8.

19. Quiles Izquierdo J, Vioque J. Prevalencia de obesidad en la Comunidad Valenciana. Med Clin (Barc) 1996;106:529-33.

20. Subdirección General de Información y Estadísticas Sanitarias. Encuesta Nacional de Salud 1993. Rev San Hig Púb 1994; 68:121-78.

21. Manson JE, Willett WC, Stampfer MJ et al. Body weight and mortality among women. $\mathrm{N}$ Eng J Med 1995; 333:677-85.

22. Manson JE, Colditz G, Stampfer MJ, Willet WC Rosner B, Monson RR et al. A prospective study of obesity and risk of coronary heart disease in women. N Eng J Med 1990; 322:882-9.

23. Registro poblacional de infartos de miocardio en la Región de Murcia: monitorización de la incidencia, la tasa de ataque, la letalidad y la mortalidad de la cardiopatía isquémica entre 1996 y 1998. Proyecto coordinado FIS n. ${ }^{\circ}$ 96/0026-04 